\title{
CUIDADOS PRÉ-OPERATÓRIOS EM HEPATOPATAS
}

\author{
Fabio Colagrossi Paes Barbosa ${ }^{*}$, Fabio Gonçalves Ferreira², Mauricio Alves Ribeiro ${ }^{3}$, Luiz Arnaldo Szutan ${ }^{4}$
}

Trabalho realizado na Irmandade Santa Casa de Misericórdia de São Paulo, São Paulo, SP

\author{
*Correspondência: \\ Irmandade Santa Casa de \\ Misericórdia de São Paulo \\ OMB - Grupo de Fígado (área II) \\ Rua Dr. Cesário Mota Jr, 112 \\ - Vila Buarque \\ São Paulo - SP \\ CEP: 01221020 \\ fabiocpb@hotmail.com
}

\begin{abstract}
RESUMO
Cirurgias realizadas em pacientes com reserva funcional hepática reduzida podem apresentar altos índices de morbi-mortalidade. Uma triagem pré-operatória ideal visa detectar a doença hepática pré-existente, sem necessidade de métodos invasivos. A história clínica e o exame físico fornecem pistas importantes. A quantificação da função hepática de rotina não é necessária, a menos que exista alguma alteração na história ou exame físico. A doença hepática causa diversos efeitos na cirurgia e anestesia. A indução anestésica, hemorragias, hipoxemia, hipotensão, drogas vasoativas e até a posição do paciente na mesa cirúrgica podem provocar diminuição na oxigenação e maior risco de disfunção hepática no intra e pós-operatório. A cirurgia de emergência é um grande preditor de mau prognóstico assim como a sepse e as reoperações. Para uma correta preparação pré-operatória, deve-se levar em consideração a natureza da doença hepática, sua gravidade e o tipo de cirurgia a ser realizada. Algumas ações devem ser tomadas no pré-operatório para diminuir as chances de complicações em pacientes portadores de hepatopatias submetidos a procedimentos cirúrgicos. 0 combate à coagulopatia, encefalopatia, ascite, disfunção renal e pulmonar, peritonite bacteriana espontânea e varizes de esôfago deve ser precoce e agressivo. Doentes Child-Pugh C e MELD > 15 não devem ser submetidos a cirurgias eletivas. Doentes Child-Pugh B e com MELD de 10 a 15 podem ser submetidos a pequenos procedimentos cirúrgicos em caso de extrema necessidade e com cautela. Finalmente doentes Child-Pugh A e MELD $<10$ podem ser operados.
\end{abstract}

UNITERMOS: Cuidados pré-operatórios. Hepatopatias. Insuficiência hepática.

\section{INTRODUÇÃO}

Cirurgias realizadas em pacientes com reserva funcional hepática reduzida podem apresentar altos índices de morbimortalidade. Garrison et al., em 1984, mostraram que $10 \%$ dos pacientes com doença hepática avançada seriam submetidos a algum tipo de tratamento cirúrgico diferente do transplante nos dois anos finais de suas vidas. ${ }^{1}$ Além disso, pacientes assintomáticos com cirrose não diagnosticada podem ser submetidos a cirurgias eletivas. Uma função hepática crítica prejudica o metabolismo das drogas, a depuração de toxinas exógenas e endógenas e a produção de proteínas plasmáticas. Dessa forma, é necessária uma avaliação pré-operatória adequada para identificar tais problemas e controlá-los, diminuindo o risco cirúrgico. ${ }^{2}$

Uma triagem pré-operatória ideal visa detectar a doença hepática pré-existente, sem necessidade de métodos invasivos. A história clínica e o exame físico fornecem pistas importantes como a presença de icterícia, ascite, spider, hepato-esplenomegalia, eritema palmar e rarefação de pelos. Fatores de risco como hemotransfusões, uso de drogas, alcoolismo, história familiar de icterícia ou doença hepática hereditária, reações adversas à anestesia e o uso de medicações devem ser questionados. ${ }^{2}$

A quantificação da função hepática de rotina não é necessária, a menos que exista alguma alteração na história ou exame físico. Apesar do grande número de pessoas infectadas pelos vírus da hepatite e da grande frequência da doença hepática gordurosa associada à obesidade, a prevalência de doença hepática na população geral permanece baixa. Um estudo envolvendo 7620 cirurgias eletivas, mostrou apenas 11 pacientes com alteração nas provas de função hepática, não justificando seu uso indiscriminado. ${ }^{3}$ Caso as provas de função hepática estejam alteradas, a cirurgia deve ser adiada para realização de uma melhor investigação. Pacientes com ALT e AST aumentadas mais de três vezes e/ou qualquer aumento

1. Pós-graduando do grupo de Cirurgia do Fígado e Hipertensão Portal da Santa Casa de São Paulo e médico assistente do grupo de Transplante Hepático da Santa Casa de São Paulo, São Paulo, SP

2. Doutor em cirurgia; médico assistente do grupo de Cirurgia do Fígado e Hipertensão Portal da Santa Casa de São Paulo; professor assistente da Faculdade de Ciências Médicas da Santa Casa de São Paulo e chefe de plantão do Serviço de Emergência da Santa Casa de São Paulo, São Paulo, SP

3. Pós-graduando do grupo de Cirurgia do Fígado e Hipertensão Portal da Santa Casa de São Paulo e chefe de plantão do Serviço de Emergência da Santa Casa de São Paulo, São Paulo, SP

4. Doutor em cirurgia; chefe do Grupo de Cirurgia do Fígado e Hipertensão Portal da Santa Casa de São Paulo; professor adjunto da Faculdade de Ciências Médicas da Santa Casa de São Paulo e diretor do curso de Medicina da Faculdade de Ciências Médicas da Santa Casa de São Paulo, São Paulo, SP 
das bilirrubinas, apresentam incidência de cirrose assintomática de 6 a $34 \% .^{4,5}$

Child e Turcotte, em $1964^{6}$, analisaram 128 pacientes submetidos à cirurgia de descompressão portal para varizes de esôfago sangrantes. Foram pesquisadas as variáveis comuns dos pacientes que evoluíram mal. Cinco variáveis apresentaram significância: desnutrição, encefalopatia, ascite, hipoalbuminemia e hiperbilirrubinemia (Tabela 1).

Dez anos depois, Pugh e Murray-Lyon ${ }^{8}$ descreveram os resultados da transecção esofágica para controle de hemorragia como ponte para descompressão portal. Pugh acrescentou o tempo de protrombina ao score e adicionou valores numéricos, entretanto, eliminou o estado nutricional. Os elementos clínicos e laboratoriais utilizados pelo score de Child-Turcotte-Pugh (CTP) avaliam as funções primárias do fígado. Todo o organismo estará em risco durante a doença hepática avançada (Tabela 2).

Outra forma de avaliação pode ser realizada por meio do Model for End-Stage Liver Disease (MELD) baseado em valores de creatinina, bilirrubina total e INR. É usado atualmente por muitos países como critério para posicionamento de pacientes em lista de transplante de fígado.

MELD $=\left[9,6 \times \log _{e}(\right.$ creatinna $\left.)\right]\left[3,8 \times \log _{e}\right.$ (bilirrubina) $][11,2$ $\left.x \log _{e}(I N R)\right] 6,4$

Obs: para cada valor abaixo de um deve-se utilizar um; o valor máximo da creatinina na fórmula é quatro; se o paciente foi dialisado duas vezes na última semana utiliza-se creatinina de quatro.

Fonte: Adaptado de Kamath et at. $(2001)^{9}$.

\begin{tabular}{|c|c|c|c|}
\hline & Classe A & Classe B & Classe C \\
\hline Nutrição & Bem nutrido & $\begin{array}{l}\text { Desnutrição } \\
\text { moderada }\end{array}$ & $\begin{array}{l}\text { Desnutrição } \\
\text { grave }\end{array}$ \\
\hline Ascite & Ausente & $\begin{array}{l}\text { Moderada } \\
\text { (controlada com } \\
\text { diuréticos) }\end{array}$ & $\begin{array}{l}\text { Grave (não } \\
\text { controlada com } \\
\text { diuréticos) }\end{array}$ \\
\hline $\begin{array}{l}\text { Encefalo- } \\
\text { patia }\end{array}$ & Ausente & Grau 1 & Graus 2 e 3 \\
\hline $\begin{array}{l}\text { Tempo de } \\
\text { protrombina }\end{array}$ & $\begin{array}{l}0-2 \text { seg > } \\
\text { controle }\end{array}$ & 2-4 seg $>$ controle & $\begin{array}{l}\geq 4 \text { seg }> \\
\text { controle }\end{array}$ \\
\hline Bilirrubina & $0-2 \mathrm{mg} / \mathrm{dl}$ & $2-3 \mathrm{mg} / \mathrm{dl}$ & $>3 \mathrm{mg} / \mathrm{dl}$ \\
\hline Albumina & $>3,5 \mathrm{~g} / \mathrm{dl}$ & $2,5-3,5 \mathrm{~g} / \mathrm{dl}$ & $<2,5 \mathrm{~g} / \mathrm{dl}$ \\
\hline
\end{tabular}

\begin{tabular}{|c|c|c|c|}
\hline \multicolumn{4}{|c|}{ Tabela 2 - Pontuação de Child-Turcotte-Pugh (CTP) } \\
\hline & 1 & 2 & 3 \\
\hline Encefalopatia & Ausente & Graus 1 e 2 & Graus 3 e 4 \\
\hline Ascite & Ausente & Leve & Moderada \\
\hline Bilirrubina & $1-2 \mathrm{mg} / \mathrm{dl}$ & $2-3 \mathrm{mg} / \mathrm{dl}$ & $>3 \mathrm{mg} / \mathrm{dl}$ \\
\hline Albumina & $>3,5 \mathrm{~g} / \mathrm{dl}$ & $2,8-3,5 \mathrm{~g} / \mathrm{dl}$ & $<2,8 \mathrm{~g} / \mathrm{dl}$ \\
\hline $\begin{array}{l}\text { Tempo de } \\
\text { protrombina } \\
\text { acima do controle }\end{array}$ & $1-4$ seg & 4-6 seg & $>6 \mathrm{seg}$ \\
\hline
\end{tabular}

\section{Efeitos da hepatopatia na cirurgia e anestesia}

A doença hepática causa diversos efeitos na cirurgia e anestesia como aumento na duração das drogas anestésicas por alteração no metabolismo do citocromo P450, menor concentração de proteínas plasmáticas e menor excreção biliar. Deve-se evitar o uso de certos opioides como a morfina no cirrótico, pelo aumento em sua biodisponibilidade e sua meia vida prolongada. O metabolismo do fentanil parece não ser afetado pela disfunção hepática. Benzodiazepínicos como midazolam e diazepam apresentam metabolismo lentificado, devendo ser evitados com o risco de precipitar encefalopatia e depressão do sistema nervoso central. ${ }^{10}$

O fluxo sanguíneo hepático, especialmente da artéria hepática, é diminuído durante a anestesia geral e cirurgia. Essa redução na oxigenação é crítica e leva a perdas das funções hepáticas mínimas existentes. Quanto aos agentes inalatórios, o halotano mostra a maior redução no fluxo hepático e pode precipitar hepatite droga-induzida. Este fluxo é mais preservado com uso do isoflurano, principalmente se a pressão sanguínea não cai abaixo de 30\%.7 A indução anestésica, hemorragias, hipoxemia, hipotensão, drogas vasoativas e até a posição do paciente na mesa cirúrgica podem provocar diminuição na oxigenação e maior risco de disfunção hepática no intra e pós-operatório. ${ }^{11}$

A cirurgia de emergência é um grande preditor de mau prognóstico assim como a sepse e as reoperações. ${ }^{7}$ Mansour et al. (1997) mostra mortalidade para cirurgias eletivas de 10\% CPT A, 30\% para B e $82 \%$ para C. Para emergência, a mortalidade foi de $22 \%$ para CPT A, 38\% para B e $100 \%$ para C. ${ }^{12}$ A colecistectomia laparoscópica é considerada segura para cirróticos compensados. ${ }^{7}$

\section{Avaliação pré-operatória}

Para uma correta preparação pré-operatória, deve-se levar em consideração a natureza da doença hepática, sua gravidade e o tipo de cirurgia a ser realizada. ${ }^{2}$

Quanto à natureza da doença hepática, a presença de icterícia obstrutiva aumenta a mortalidade perioperatória, porém, a drenagem pré-operatória da via biliar não melhora a morbimortalidade, a não ser em caso de colangite, quando a drenagem deve ser realizada. A presença de hepatite aguda aumenta a morbi-mortalidade da cirurgia. As cirurgias eletivas devem ser suspensas, da mesma forma que na presença de hepatite alcoólica aguda. Hepatite crônica em doentes assintomáticos não é contraindicação cirúrgica, desde que não haja disfunção hepática. Na presença de cirrose devem ser avaliadas a função hepática, presença de hipertensão portal, ascite, disfunção renal e hipertensão porto-pulmonar. ${ }^{13}$

A gravidade da doença hepática pode ser avaliada pela classificação de Child-Turcotte-Pugh. Cirróticos submetidos a cirurgias abdominais possuem mortalidade de 10\%, 30\% a $31 \%, 76 \%$ a $82 \%$ para CTP A, B e C respectivamente. A acurácia é limitada pela subjetividade dos parâmetros clínicos. ${ }^{14}$ Outra forma de avaliação pode ser realizada por meio do MELD baseado em valores de creatinina, bilirrubina total e INR. Em cirróticos submetidos à colecistectomia, o MELD e CTP foram bom preditores de morbidade pós-operatória (10\% para MELD 8 e 44\% se acima de 8). ${ }^{15}$ Testes da função hepática como a retenção do verde de indocianina, taxa de absorção hepática 
de galactosil albumina sérica humana conjugada ao tecnécio99m(99mTc-GSA) e a mensuração do metabólito da lidocaína monoetil-glicina-xilidida são raramente utilizados.

O tipo de cirurgia influi no resultado pós-operatório. Cirurgias de emergência têm alta mortalidade no hepatopata. O risco também é aumentado nas cirurgias eletivas abdominais e cardíacas. Cirurgia cardíaca com by pass cardiopulmonar teve mortalidade 0\% em CTP A, 50\% em CTP B e 100\% em CTP C. ${ }^{16}$ Em geral, o risco para cirurgias urológicas e ortopédicas é menor que para cirurgias abdominais e cardíacas.

Após uma avaliação pré-operatória cuidadosa, cirróticos CTP A podem ser submetidos a cirurgias eletivas. Porém, cirróticos CTP B não devem ser submetidos a ressecções hepáticas ou cirurgias cardíacas, mas podem realizar outras cirurgias após otimização de sua condição clínica. Quanto aos cirróticos CTP C, não é recomendada a realização de nenhum tipo de procedimento cirúrgico. Uma vantagem da utilização da escala MELD é o fato de ser uma escala contínua, que pode ser realizada repetidamente. Ao ser reduzida, indica compensação do paciente, podendo-se então recomendar realização de procedimento cirúrgico quando o MELD está abaixo de 10. Quando a escala MELD estiver entre 10 e 15, deve-se ter cautela para realizar cirurgias, que devem ser indicadas somente em casos estritamente necessários. Porém, em doentes com MELD acima de 15, cirurgias são contraindicadas. A presença de hipertensão portal (varizes de esôfago, ascite, esplenomegalia com plaquetopenia) deve ser avaliada, pois é um fator preditor de pior prognóstico que os critérios de CTP em pacientes submetidos a ressecções hepáticas. ${ }^{17}$

\section{Correção de distúrbios}

Após uma avaliação adequada, algumas ações devem ser tomadas no pré-operatório para diminuir as chances de complicações em pacientes portadores de hepatopatias submetidos a procedimentos cirúrgicos.

A coagulopatia existente nesses doentes possui etiologia multifatorial como a desnutrição, má absorção de nutrientes, colestase e diminuição na síntese proteica. Com exceção do fator de Von Willebrand, os fatores da coagulação e a maioria dos seus inibidores são produzidos no fígado. Além da baixa concentração plasmática dos fatores de coagulação, o hiperesplenismo e a trombocitopenia também podem estar presentes e contribuir para coagulopatia. Deve-se administrar vitamina $\mathrm{K}$ intramuscular ou endovenosa por três dias e plasma fresco congelado (PFC) se necessário. Essas medidas podem não ser suficientes nas coagulopatias graves e a hipervolemia causada pelo PFC pode ser prejudicial, causando congestão pulmonar. A monitorização da pressão venosa central é essencial, sendo necessário uso de diuréticos nesses casos. 0 crioprecipitado pode ser infundido com um volume menor e ainda possui grande quantidade de fibrinogênio e fator de Von Willebrand. O uso do fator VIla recombinante é seguro e efetivo em cirróticos, porém sua meia vida é curta, pouco disponível e altamente custoso. Deve-se realizar transfusão de plaquetas em pré-operatório quando estas estão abaixo de 50000. A coagulopatia pode ser monitorada no intraoperatório por meio do tromboelastograma. Doentes com intensa atividade fibrinolítica secundária a perda de sangue maciça, podem se beneficiar do ácido épsilon-amino-caproico assim como ácido transanêmico, pois ambos apresentam atividade antifibrinolítica. ${ }^{18,19}$
A ascite deve ser tratada no pré-operatório para evitar complicações respiratórias e deiscência da ferida no período pósoperatório. Restrição de sódio na dieta e uso de diuréticos por via oral (espironolactona/ furosemida) fazem parte do tratamento. Em casos de aumento do nível de creatinina e sódio sérico abaixo de $125 \mathrm{mEq} / \mathrm{l}$, os diuréticos devem ser interrompidos. Deve-se manter especial atenção com o controle dos eletrólitos no préoperatório. Uma dieta ocidental típica contém cerca de $150 \mathrm{mmol}$ de sódio por dia, do qual 15\% é proveniente de sal adicionado ao alimento e $70 \%$ origina-se dos próprios alimentos. A restrição do sal deve se manter em torno de 90mmol/dia (2g de sódio), conseguida com uma dieta sem sal de adição e evitando-se alimentos industrializados. ${ }^{20}$

É comum a presença de hiponatremia no cirrótico, por isso é recomendado restrição da ingestão de líquidos (para pacientes euvolêmicos com função renal normal e sódio sérico $<130 \mathrm{mEq} / \mathrm{l}$ ) balanço hídrico, medida diária da circunferência abdominal assim como pesagem. Paracentese de alívio deve ser realizada em caso de restrição ventilatória. Albumina venosa deve ser administrada em caso de paracenteses acima de 5 litros (8g de albumina por litro de ascite) e deve-se evitar a hiper-hidratação no pós-operatório. ${ }^{20,21}$

A encefalopatia pode estar presente. A principal atitude consiste em reverter os fatores precipitantes (hipocalemia, alcalose, hipoglicemia, hipovolemia, hemorragia digestiva, medicações e infecções). A lactulose oral diminui o ph do intestino facilitando a conversão da amônia em íons amônio que não são absorvidos. Antibióticos por via oral como o sulfato de neomicina e posteriormente outros antibióticos orais pouco absorvíveis passaram a ser utilizados na prática clínica com a intenção de esterilizar os cólons impedindo a formação de amônia e/ou produtos nitrogenados. Em casos crônicos, que necessitem de tratamentos prolongados, não se indica o uso de neomicina devido efeitos colaterais importantes, como nefrotoxicidade e/ou ototoxicidade. A rifaximina, antibiótico sintético, relacionado à rifamicina com baixa absorção sistêmica tem sido indicada. ${ }^{22} \mathrm{O}$ clister de neomicina também diminui a população bacteriana produtora de amônia no intestino. Não há consenso quanto à suplementação de zinco. Não se indica restrição proteica para profilaxia da encefalopatia hepática, a maioria dos doentes tolera bem a proteína dietética até cerca de $1,75 \mathrm{~g} / \mathrm{Kg}$ de peso por dia. Em pacientes com encefalopatia instalada há benefício de dietas enriquecidas com aminoácidos de cadeia ramificada em detrimento de aminoácidos de cadeia aromática. ${ }^{23}$

A encefalopatia pode variar de uma confusão mental até 0 coma. Controlar o aumento da pressão intracraniana que leva ao coma e à morte encefálica com medidas como elevação da cabeceira, intubação orotraqueal com hiperventilação, diuréticos osmóticos, plasmaférese e eventualmente exanguíneo-transfusão. O flumazenil, antagonista benzodiazepínico, pode produzir uma melhora nas manifestações neurológicas assim como o agonista dopaminérgico bromocriptina, porém, a longo prazo, não previnem o coma. Atualmente utiliza-se o L-aspartato-I-ornitina para remoção da amônia do fígado e músculos. Um envelope duas vezes ao dia ou até oito ampolas diárias. Exerce sua ação nos ciclos metabólicos do fígado, particularmente no ciclo da ureia, diminuindo rapidamente os níveis sanguíneos elevados de amônia. A capacidade de detoxificação, particularmente do ciclo da uréia, é aumentada e o balanço energético é melhorado. ${ }^{24}$ 
Pacientes hepatopatas comumente possuem disfunção renal. A síndrome hepato-renal pode estar relacionada a nefrotoxinas não depuradas pelo fígado debilitado e a alterações na perfusão renal secundária a aumento na pressão intra-abdominal em pacientes com ascite volumosa. Apresenta-se como insuficiência renal oligúrica. Resolve-se imediatamente após o transplante hepático. Anteriormente ao transplante o tratamento se dá por meio de diálise e hemofiltração, paracenteses de alívio e infusão de albumina e terlipressina. Evita-se o uso de anti-inflamatórios e aminoglicosídeos. ${ }^{25}$

A disfunção pulmonar caracterizada por uma tríade clínica: doença hepática; alteração da troca gasosa pulmonar com elevação do gradiente alvéolo-arterial de oxigênio ( $\mathrm{AaO} 2)$ com ou sem hipoxemia; e dilatações vasculares intrapulmonares leva a suspeita da síndrome hepato-pulmonar. ${ }^{26}$ Não há terapia efetiva para esta síndrome, suplementação de oxigênio e suporte clínico são utilizados até que o paciente consiga um transplante de fígado. Tem um prognóstico muito ruim. Ortodeóxia e platipneia (dessaturação e encurtamento da respiração em posição ereta comparada ao decúbito dorsal horizontal) são os sinais clínicos mais importantes. ${ }^{27}$ Pacientes que apresentam melhora clínica com uso de vasodilatadores antes do transplante possuem melhor prognóstico. A descompressão com TIPS (transjugular intrahepatic portosystemic shunt) pode melhorar a oxigenação arterial, diminuindo os shunts pulmonares. Pacientes que sobrevivem ao transplante de fígado recuperam as funções pulmonares. ${ }^{28}$

A desnutrição é muito comum em hepatopatas. Pacientes CTP C possuem depleção de proteínas, excesso de água e hipermetabolismo. A reserva de glicogênio hepático e muscular está depletada e o paciente possui risco de hipoglicemia no perioperatório. Por outro lado, pacientes com esteatohepatite não alcoólica apresentam resistência à insulina e podem apresentar hiperglicemia. Esses pacientes devem receber suporte enteral e parenteral hiperglicídico e hiperlipídico com restrição de aminoácidos, principalmente os aromáticos, para se evitar a encefalopatia. Suplemento de vitamina B deve ser oferecido no pré-operatório. ${ }^{29}$

Os sangramentos de varizes esofágicas podem ser controlados por meio de shunts entre o sistema venoso portal e o sistema de retorno pela veia cava inferior. Esses shunts podem ser cirúrgicos (meso-cava, espleno-renal, porto-cava) ou percutâneos (TIPS). ${ }^{30}$ Análogos da somatostatina têm sido utilizados no controle do sangramento de pacientes com gastropatia e varizes de cólon. ${ }^{31,32}$ $\mathrm{O}$ beta bloqueador deve ser usado na profilaxia do sangramento. $\mathrm{A}$ mortalidade em doentes com varizes esofágicas sangrantes é alta: $42 \%$ para TIPS e 79\% para transecção esofágica. O ressangramento ocorreu em 15,6\% dos que utilizaram TIPS e em 26,2\% dos submetidos a transecção esofágica. Portadores de varizes de esôfago que serão submetidos a procedimentos cirúrgicos devem realizar tratamento endoscópico das varizes (escleroterapia ou ligadura elástica) no pré-operatório pelo risco de descompensação e sangramento associados ao ato cirúrgico. ${ }^{33}$

Os hepatopatas comumente possuem doença cardiovascular. Trata-se de doença hiperdinâmica, com índice cardíaco duas vezes o normal, baixa resistência vascular periférica e pressão arterial pouco diminuída. Pacientes com isquemia miocárdica induzida e hepatopatas devem ser submetidos a revascularização do miocárdio primeiro e depois ao transplante de fígado. ${ }^{7}$

A peritonite bacteriana espontânea é normalmente causada por germes Gram positivos. Em doentes hospitalizados é comum a infecção por Gram negativos como E. coli e Klebsiella. Antibioticoprofilaxia é recomendada com cefalosporina de terceira geração ou quinolonas em ascíticos com hemorragia digestiva. ${ }^{34}$

A esteatohepatite não alcoólica, cada vez mais frequente na população, pode estar associada a obesidade, diabetes tipo II hipertrigliceridemia e esteatose hepática. Pode progredir para cirrose e falência hepática. O IMC não têm relação com a presença de lesão hepática significante. Biópsia hepática de rotina deve ser realizada durante as cirurgias de obesidade. Doentes assintomáticos com esteatose devem realizar biópsia do fígado caso haja alterações nas provas de função ou lesão hepáticas. $^{7}$

Hiponatremia severa ocorre em hepatopatas avançados devido à retenção anormal de água e função renal prejudicada. Leva a anormalidades no funcionamento do sistema nervoso central. A correção da hiponatremia muito rápida pode levar à mielinólise pontina central, por isso o sódio deve ser corrigido lentamente. Em pacientes assintomáticos, apenas a restrição hídrica protege contra o edema cerebral. Em caso de sintomas neurológicos, deve-se administras $\mathrm{NaCl} 3 \%$ intravenoso lentamente. ${ }^{35}$

Alguns casos específicos devem ser levados em consideração. Etilistas têm maior risco de hepatotoxicidade por acetaminofen, por isso, é necessário cuidado na analgesia. Na doença hepática auto imune, usuário de prednisona devem receber doses de hidrocortisona venosa no intraoperatório. Doentes com hemocromatose devem ser avaliados para diabetes e cardiomiopatia no pré-operatório. Pacientes com doença de Wilson devem ter a dose de penicilamina diminuída uma a duas semanas antes da cirurgia. ${ }^{2}$

\section{Quem e quando operar?}

Hanje e Patel $(2007)^{2}$ propuseram uma sequência para conduta cirúrgica em hepatopata:

Doentes com hepatite aguda devem ter a cirurgia suspensa até melhora clínica e laboratorial.

Doentes com hepatite aguda fulminante são candidatos a transplante de fígado.

Doentes com hepatite crônica não cirróticos podem ser submetidos a cirurgias.

Doentes com hepatite crônica com cirrose ou suspeita de cirrose deve-se considerar o MELD e a classificação de CTP:

a. MELD > 15 ou CTP C: não devem ser operados.

b. MELD de 10 a 15 ou CTP B: podem ser realizadas pequenas cirurgias em caso de extrema necessidade e com muita cautela.

c. MELD < 10 ou CTP A: podem ser operados.

Kanzler et al. (2007) afirmam que em cirróticos, somente pacientes CTP A com bilirrubinas normais e sem hipertensão portal, devem ser candidatos a ressecções hepáticas. ${ }^{36}$ Para Yamagiwa et al.(1997), as cirurgias eletivas são possíveis em pacientes CTP A, cirurgias com pouca perda sanguínea são possíveis em pacientes CTP $B$, porém em CTP $C$ apenas casos de emergência devem ser operados. Os limites para cirurgia em cirróticos são a retenção do verde de indocianina $\geq 0.04 / \mathrm{min}$, bilirrubina total $\leq 3 \mathrm{mg} / \mathrm{dl}, \mathrm{AP}>50 \%$ e R $15 \geq 40 \% .{ }^{37}$ 


\section{Conclusão}

Diversas cirurgias são possíveis de se realizar no paciente hepatopata com segurança. É preciso avaliação adequada da função hepática, quantificação do porte da cirurgia, cuidados com a anestesia e vigilância no pós-operatório para se obter um resultado com morbimortalidade aceitável.

\section{Conflito de interesse: não há}

\section{SUMMARY}

\section{Pre-operative care for liver disease patients}

Patients with impaired hepatic functional reserve when submitted to surgeries may have high rates of morbidity and mortality. Preexisting liver disease should be detected without need for invasive methods. Clinical history and physical examination provide important clues. Laboratory liver function is not necessary unless there are changes in history or physical examination. Liver disease has many effects on surgery and anesthesia. A decrease in oxygenation and increased risk of liver dysfunction can be caused by anesthesia, hemorrhage, hypoxemia, hypotension, vasoactive drugs or the patient's position on the operating table during and after surgery. Emergency surgery is a major predictor of poor prognosis as well as sepsis and reoperations. The nature of liver disease, severity and type of surgery to be performed should take into account for a correct preoperative preparation. Some actions must be taken at preoperative to decrease chances of complications in patients with liver disease undergoing surgical procedures. Very close attention should be given to coagulopathy, encephalopathy, ascites, renal and pulmonary dysfunction, spontaneous bacterial peritonitis and esophageal varices. Patients with Child-Pugh score C and MELD $>15$ should not undergo elective surgery. Patients with Child-Pugh score $B$ and MELD 10 to 15 may undergo minor surgical procedures with care in cases of extreme necessity. Patients with Child-Pugh score $A$ and $M E L D<10$ may be submitted to elective surgery. [Rev Assoc Med Bras 2010; 56(2): 222-6]

KEY WORDS: Preoperative care. Liver diseases. Liver failure.

\section{REFERÊNCIAS}

1. Garrison RN, Cryer HM, Howard DA, Polk HC Jr. Clarification of risk factors for abdominal operations in patients with hepatic cirrhosis. Ann Surg. 1984;199:648-55.

2. Hanje AJ, Patel T. Preoperative evaluation of patients with liver disease. Gastrol Hepatol. 2007;4:266-76.

3. Schemel WH. Unexpected hepatic dysfunction found by multiple laboratory screening. Anesth Analg. 1976;55:810-12

4. Hay JE, Czaja AJ, Rakela J, Ludwig J. The nature of unexplained chronic aminotransferase elevations of a mild to moderate degree in asymptomatic patients. Hepatology. 1989;9:193-97.

5. Hultcrantz R, Glaumann H, Lindberg G, Nilsson LH. Liver investigation in 149 asymptomatic patients with moderately elevated activities of serum aminotransferases. Scand J Gastroenterol. 1986;21:109-13.

6. Child CG, Turcotte JG. Surgery and portal hypertension. In: Child CG, editor. The liver and portal hypertension. Philadelphia: Saunders; 1964. v. 1, p.1-84.

7. Wiklund RA. Preoperative preparation of patients with advanced liver disease. Crit Care Med. 2004;32:106-15.

8. Pugh R, Murray-Lyon I. Transection of the esophagus in bleeding esophageal varices. Br J Surg. 1973;60:646-52

9. Kamath PS, Wiesner RH, Malinchoc M, Kremers W, Therneau TM, Kosberg $\mathrm{CL}$, et al. A model to predict survival in patients with end-stage liver disease. Hepatology. 2001;33:464-70.
10. Delco F, Tchambaz L, Schlienger R, Drewe J, Krähenbuhl S. Dose adjustment in patients with liver disease. Drug Saf. 2005;28:529-45.

11. Kenna JG. Immunoallergic drug-induced hepatitis: lessons from halothane. J Hepatol. 1997;26:5-12.

12. MansourA, Watson W, Shayani V, Pickleman J. Abdominal operations in patients with cirrhosis: still a major surgical challenge. Surgery. 1997;122:730-6.

13. Lai EC, Chu KM, Lo CY, Mok FP, Fan ST, Lo CM, Wong J. Surgery for malignant obstructive jaundice: analysis of mortality. Surgery. 1992;112:891-6.

14. Francoz C, Durand F. The risk of surgery in patients with cirrhosis. Acta Gastroenterol Belg. 2008; 71:42-6.

15. Perkins $L$, Jeffries $M$, Patel T. Utility of preoperative scores for predicting morbidity after cholecystectomy in patients with cirrhosis. Clin Gastroenterol Hepatol. 2004;2:1123-8

16. Hayashida N, Shoujima T, Teshima H, Yokokura Y, Takagi K, Tomoeda H, et al. Clinical outcome after cardiac operations in patients with cirrhosis. Ann Thorac Surg. 2004;77:500-05.

17. Bruix J, Sherman M. Practice Guidelines Committee, American Association for the Study of Liver Diseases. Hepatology. 2005;42:1208-36.

18. French CJ, Bellomo R, Angus P. Cryoprecipitate for the correction of coagulopathy associated with liver disease. Anaesth Intensive Care. 2003;31:357-61.

19. Porte RJ, Molenaar IQ, Begliomini B, Groenland TH, Januszkiewicz A, Lindgren $\mathrm{L}$, et al. Aprotinin and transfusion requirements in orthotopic liver transplantation: a multicentre randomised double-blind study. EMSALT Study Group. Lancet. 2000;355:1303-9.

20. Moore KP, Aithal GP. Guidelines on the management of ascites in cirrhosis. Gut. 2006;55(Suppl 6):vi1-vi12.

21. Runyon BA. Management of adult patients with ascites due to cirrhosis. Practice Guidelines Committee, American Association for the Study of Liver Diseases (AASLD). Hepatology. 2004;39:841-56.

22. Strauss E. Encefalopatia hepática - atualização terapêutica. Gaz Med Bahia 2006;76(Supl 1):S43-S5.

23. Schulz GJ, Campos AC, Coelho JC. The role of nutrition in hepatic encephalopathy. Curr Opin Clin Nutr Metab Care. 2008;11:275-80.

24. Blei AT, Córdoba J. Practice Parameters Committee of the American College of Gastroenterology. Hepatic encephalopathy. Am J Gastroenterol. 2001;96:1968-76.

25. Moreau R, Lebrec D. Diagnosis and treatment of acute renal failure in patients with cirrhosis. Best Pract Res Clin Gastroenterol. 2007;21:111-23.

26. Ferreira PP, Zollinger CC, Bittencourt PL. Síndrome hepatopulmonar. GED. 2008;27(1):5-13.

27. Hoeper MM, Krowka MJ, Strassburg CP. Portopulmonary hypertension and hepatopulmonary syndrome. Lancet. 2004;363:1461-8.

28. Mortier E, Ongenae M, Poelaert J, Den Blauwen N, Decruyenaere J, Van Aken $\mathrm{J}$, et al. Rapidly progressive pulmonary artery hypertension and end-stage liver disease. Acta Anaesthesiol Scand. 1996;40:126-9

29. Weimann A, Kuse ER, Bechstein WO, Neuberger JM, Plauth M, Pichlmayr R. Perioperative parenteral and enteral nutrition for patients undergoing orthotopic liver transplantation. Results of a questionnaire from 16 European transplant units. Transpl Int1998;11(1):289-291.

30. Orozco H, Mercado MA, Chan C, Guillén-Navarro E, López-Martínez LM. A comparative study of the elective treatment of variceal hemorrhage with beta-blockers, transendoscopic sclerotherapy, and surgery. A prospective, controlled, and randomized trial during 10 years. Ann Surg2000;232:216-19.

31. Meier R, Wettstein AR. Treatment of acute nonvariceal upper gastrointestinal hemorrhage. Digestion1999; 60:47-52.

32. BJ, Riley JW. Control of colonic variceal haemorrhage with a somatostatin analogue. J Gastroenterol Hepatol1996;11:305-06.

33. Jalan R, Elton RA, Redhead DN, Finlayson ND, Hayes PC. Analysis of prognostic variables in the prediction of mortality, shunt failure, variceal rebleeding and encephalopathy following the transjugular intrahepatic portosystemic stent shunt for variceal haemorrhage. J Hepatol1995;23:123-28.

34. Jansen PL. Spontaneous bacterial peritonitis. Detection, treatment and prophylaxis in patients with liver cirrhosis. Neth J Med1997;51:123-28.

35. Lampl C, Yazdi K. Central pontine myelinolysis. Eur Neurol2002;47:3-10.

36. Kanzler S, Teufel A, Galle PR. Liver function test to predict hepatic failure after liver resection--expensive and without clinical relevance? Zentralbl Chir 2007;132(4):267-73.

37. Yamagiwa K, Kawarada Y. Preoperative estimation of liver injury and operative risk. Nippon Geka Gakkai Zasshi 1997;98(8):658-62. 